\title{
La publicidad en el marco de la comunicación para el desarrollo: hacia un nuevo modelo de publicidad para el cambio social
}

\author{
M. ${ }^{\mathrm{a}}$ Cruz Alvarado LÓPEZ*
}

Propuesto: 15 de abril de 2012

Evaluado: 23 de abril de 2012

Aceptado: 3 de mayo de 2012

(Abstracts y palabras clave al final del texto)

\section{INTRODUCCIÓN}

Mucho se ha escrito e investigado, y desde las más diversas perspectivas, sobre el papel que ha venido ejerciendo la comunicación en las distintas etapas de lo que sucesivamente, desde los años cincuenta del pasado siglo hasta el momento actual, se ha ido entendiendo como desarrollo.

Así, en la valiosa y extensa literatura que hoy existe sobre el tema, ya sea desde un punto de vista teórico (autores y textos que han tratado de sentar las bases teóricas de los paradigmas desde los que se ha entendido el desarrollo), pero también práctico (en los informes de los programas que se han llevado a cabo sobre el terreno y en los casos que han servido como objeto de análisis y cuyo conocimiento constituye una base esencial para la mejora de las acciones futuras), la comunicación ha ocupado siempre un lugar, ya sea de un modo implícito o explícito. Queremos decir que, incluso cuando no se menciona o no se incluye a la comunicación de forma directa como una herramienta de desarrollo, la comunicación está presente, aunque solo sea por el hecho de que, en algún momento, habrá que comunicarse con otros para darles a conocer nuestra teoría, nuestras ideas o nuestro proyecto. Y, si la comunicación no funciona será muy difícil que esa teoría trascienda a la comunidad científica, o que un determinado proyecto de cambio funcione.

Porque como muy bien ha sabido explicar Alfonso Gumucio, la comunicación es siempre clave para el éxito o el fracaso de un proyecto de cambio social, ya que, aunque a menudo no seamos conscientes de ello, interviene a través de sus distintas modalidades y medios en todos los niveles (y en todos los agentes) de los que depende el éxito de un programa de desarrollo: reuniones, diálogo, seminarios, publicación de noticias, elaboración y difusión de documentos y mensajes para y/o por la comunidad, etc. ${ }^{1}$.

\footnotetext{
* Universidad de Valladolid

1 Uno de los mayores expertos actuales en el tema, Alfonso Gumucio, en una de sus conocidas presentaciones, puso de manifiesto a través de unos esquemáticos dibujos, las enormes diferencias que suelen exis-
} 
Pero ¿qué consideración se ha hecho en concreto de la comunicación publicitaria en este marco?

Es el objetivo principal de este trabajo el hacer una primera aproximación al esclarecimiento de ese papel. Saber qué consideración se le ha otorgado a la publicidad en los distintos modelos teóricos de la comunicación para el desarrollo, cómo se ha utilizado sobre el terreno (si es que se ha hecho), por qué ha sido así y qué opciones de futuro hay para ella.

Partimos aquí de la idea de que el papel que la comunicación publicitaria haya podido ejercer en este marco ha quedado desdibujado, ya sea por su vinculación directa con los modelos y teorías de la modernización, hoy cuestionados; o por la existencia de determinados prejuicios negativos en relación a la publicidad como propiciadora o cómplice de algunos de los males del sistema que nos ha llevado a la situación de crisis y desigualdad actual.

Sin embargo, desde un convencimiento total de la importancia de la comunicación en los procesos de cambio social consideramos que, en particular, la publicidad no puede estar al margen de los retos de mejora que tenemos por delante.

Así, primero intentaremos ofrecer una doble perspectiva sobre la relación publicidad-desarrollo: cómo se ha entendido la publicidad desde la comunicación para el desarrollo y cómo éste ha sido una preocupación frecuente para la publicidad y para su sistema (esclarecer en definitiva cual ha sido su contribución al cambio social); para, después, esbozar el que sería un nuevo modelo desde el que poner en valor esta herramienta que, como ya se ha dicho, creemos esencial en la comunicación para el cambio social de futuro.

\section{LA PUBLICIDAD EN LA COMUNICACIÓN PARA EL DESARROLLO}

Parece oportuno iniciar este recorrido tratando de hacer algunas consideraciones sobre cómo ha sido entendida la comunicación publicitaria desde la comunicación para el desarrollo.

En primer lugar, hay que decir que la publicidad ha sido sobre todo, y es todavía en su utilización mayoritaria (tanto por parte de las entidades que trabajan en el sector del desarrollo, como de las instituciones que realizan todo tipo de acción social en los países desarrollados), un instrumento del llamado marketing social.

Esto se debe a que el origen mismo de la que hoy llamamos publicidad social, entendida como modalidad publicitaria que sirve a causas sociales, está en parte, en

tir en la práctica entre lo que una comunidad necesita y lo que a través de un proyecto de desarrollo finalmente se consigue. Ya que cada uno de los mediadores que interviene en un determinado proyecto comunitario (el financiador, los analistas, los planificadores del programa, etc.), entiende de un modo distinto la necesidad real que plantea la comunidad, y, en consecuencia, lo que hay que hacer para satisfacerla. Un problema generado por infravalorar la importancia de la comunicación en toda la cadena que, probablemente sea una de las causas de los fracasos evidentes en la historia del desarrollo. Ver en Gumucio, A. (1999): Comunicación, desarrollo y participación, presentación disponible on line en

$\mathrm{http}: / /$ www.slideshare.net/Gumucio/comunicacin-desarrollo-y-participacin, [consultada el 15 de febrero de 2012] 
la utilización que se realizó de ella como parte de los primeros modelos de desarrollo basados en las teorías de la modernización. Veamos cómo fue esta utilización, así como lo que sucedió más adelante, con el cambio de paradigma que entendía el desarrollo en términos de participación.

Desde estas teorías se creía que una de las claves del desarrollo era la transferencia de información y conocimiento, por lo que era necesario un potente mecanismo persuasivo para propiciar la expansión de los mercados y la difusión de innovaciones y tecnologías, así como su asimilación por parte de poblaciones masivas. Por ello, fundamentalmente dentro de los modelos de "difusión de innovaciones", "mercadeo social" y "promoción de la salud", basados en mayor o menor medida en una noción jerárquica y exógena del desarrollo, la comunicación publicitaria se convirtió muchas veces en prioritaria.

Entendida como un sistema ya consolidado, que había resultado eficaz durante décadas al servicio de todo tipo de causas comerciales, y de la mano de los medios de difusión masivos, la publicidad que hoy etiquetamos como tradicional, resultó imprescindible en numerosos programas de desarrollo planificados sobre todo desde el enfoque del marketing social.

Como lo sigue siendo, por ejemplo, en el campo de la salud. Así lo evidencian las numerosas y sistemáticas campañas, llevadas a cabo desde 1987 en todo el mundo, para combatir la pandemia del SIDA por recomendación de la Organización Mundial de la Salud, a las que debemos los primeros y polémicos anuncios que trataron de alertar a la población de los países desarrollados sobre las vías de contagio, las causas y las dramáticas consecuencias de la enfermedad.

Si en los modelos derivados de las teorías de la modernización, la publicidad, con su sistema tradicional también jerárquico y vertical basado en el imperativo de eficacia y en la consecución de objetivos previamente establecidos y resultados cuantificables, parecía encajar a la perfección, ¿Qué consideración se le dio desde las teorías de la dependencia?

Por lógica, si los modelos basados en las teorías de la dependencia coinciden en su crítica hacia los medios masivos y en la necesidad de desarrollar, de modo específico, un sentido crítico hacia estos, a priori no parece que la publicidad tradicional sea contemplada como uno de sus recursos.

Y más si tenemos en cuenta el sustrato ideológico desde el que se gestaron los movimientos políticos y sociales que favorecieron las luchas por la independencia o contra las dictaduras en África, Asia y América Latina, ya de por sí enormemente crítico con el sistema capitalista dominante y, por ende, con la publicidad.

Es en éste último contexto, el de América Latina, en el que durante las cuatro últimas décadas se van a establecer poco a poco y con brillantísimas aportaciones los cimientos teóricos de los nuevos modelos.

Pero, como suele suceder en este ámbito, mucho antes de esta teorización van a surgir las primeras experiencias (hoy consideradas paradigmáticas) de un modelo alternativo de comunicación en relación al desarrollo, que anticipan las bases de lo que van a ser después ${ }^{2}$ : el modelo de "comunicación para el desarrollo" pro-

\footnotetext{
2 Nos referimos a casos bien documentados como los de las Radioescuelas de Colombia y las Radios mineras de Bolivia, que tuvieron lugar entre el último tercio de la década de 1940 y el primero de la de 1950.
} 
piamente dicho, el de la "comunicación alternativa y comunitaria" y el de la "comunicación participativa"; pero también el más reciente de la "comunicación para el cambio social".

Desde todos ellos, la publicidad tradicional quedaría prácticamente fuera ya que en general, se entiende que en los procesos de cambio social la comunicación no puede ser instrumental, vertical, unidireccional, impuesta y masiva sino verdadera comunicación. Es decir: democrática, horizontal, dialógica, respetuosa con la diversidad cultural e interpersonal; y que sólo la comunicación participativa es una apuesta ética en este terreno.

Si además tenemos en cuenta que el problema del desarrollo ya no se plantea en términos de transferencia exógena de conocimiento o de cambio de actitudes y comportamientos, sino de desigualdades estructurales, el papel reservado a la comunicación y específicamente a la publicidad, tiene que ser necesariamente otro.

Además, se parte también una consideración crítica (a veces convertida en prejuicio negativo) fundamentada en el hecho de que la publicidad haya formado parte de numerosos programas de desarrollo que hoy pueden considerarse verdaderos fracasos. Programas a menudo encabezados por organismos internacionales, que con importantes presupuestos, no fueron sostenibles o incluso llegaron a perjudicar a sus potenciales beneficiarios. Programas en los que el desconocimiento y la ignorancia por parte de los planificadores de la cultura y diversidad locales, la existencia de intereses ajenos al beneficio de la comunidad, el arraigo en el modelo difusionista (con las implicaciones que ello tiene), y la consideración instrumental y no democrática del papel de la comunicación (y por tanto de la publicidad); resultaron grandes decepciones.

Y la difusión masiva de mensajes publicitarios formó parte de ellas. Por ejemplo, en las campañas de salud que a través de los medios masivos proponían insistentemente a las mujeres de zonas poco desarrolladas "hervir el agua", sin tener en cuenta las dificultades existentes en muchos casos para conseguir leña para hacer un simple fuego. $\mathrm{O}$ en los carteles que formaban parte de una campaña de promoción de la salud llevada a cabo en Papúa Nueva Guinea, en los que para concienciar a la población contra el tabaco se habían representado cigarrillos con filtro occidentales, detallándose todos los componentes dañinos que contenían, cuando la mayoría de la población de Papúa Nueva Guinea fumaba cigarrillos artesanales ${ }^{3}$.

También el término campaña, que sigue siendo empleado habitualmente en la profesión publicitaria, así como todo lo que implica (acciones y estrategias de comu-

\footnotetext{
Ver por ejemplo en: Vaca Gutiérrez, H. (2011), "Procesos interactivos mediáticos de Radio Sutatenza con los campesinos de Colombia (1947-1989)", en Signo y Pensamiento, $\mathrm{n}^{\circ}$ 58, Documentos de Investigación, pp.254-269, volumen 30, enero-junio 2011, disponible en

http://recursostic.javeriana.edu.co/cyl/syp/components/com_booklibrary/ebooks/5818Vaca.pdf [consultado el 10 de febrero de 2011]; Aguirre Alvis, J.L., y Condori, M.(2000), Las radios comunitarias en Bolivia: despojados de todo, incluso de la palabra, Presencia Juvenil, la Paz, Bolivia.

3 Este y otros casos están recogidos en Gumucio Dragón, A. (2004), "El cuarto mosquetero: la comunicación para el cambio social", en Investigación y Desarrollo, col. 12, n ${ }^{\circ} 1$ (2004) pp. 2-23. (Documento presentado al VI congreso de la Asociación latinoamericana de Investigadores de la Comunicación- ALAIC, realizado en Santa Cruz de la Sierra, Bolivia, del 5 al 8 de junio de 2002). Disponible en http://ciruelo.uninorte.edu.co/pdf/invest_desarrollo/12-1/el_cuarto_mosquetero.pdf [consultada el 10 de octubre de 2011]
} 
nicación costosas y limitadas en el tiempo, impuestas desde fuera y diseñadas sin tener en cuenta las opiniones de la comunidad) es rechazado desde los modelos dialógico y participativo, ya que en ellos se consideran prioritarios los procesos de participación democrática de la comunidad para el diseño de la estrategia de comunicación necesaria en cada caso.

En el caso del modelo específicamente llamado de "comunicación para el desarrollo", la publicidad tradicional puede tener cabida ya que desde el mismo sí se entiende que pueda ser necesario de forma específica el uso de los medios masivos, sobre todo para fomentar la participación de la comunidad en un determinado programa.

Sin embargo, para poder servir como herramienta en los modelos alternativo y participativo y también al modelo de "comunicación para el cambio social", la publicidad como sistema tendría que transformarse $\mathrm{y}$, más allá de una publicidad social tendría que gestarse otra publicidad. Algo que, como veremos después, dados los enormes cambios por los que esta modalidad comunicativa está pasando no creemos imposible.

De hecho, según afirma Alfonso Gumucio en el prólogo del texto Haciendo Olas: "Lo extraordinario en la comunicación participativa es que puede adoptar diferentes formas, de acuerdo a las necesidades, y que no es posible imponer un modelo único sobre la riqueza de perspectivas y de interacciones culturales" 4 . Y una de esas formas podría ser, por qué no, la publicidad tal y como después la vamos a entender.

Desde los modelos dialógico y participativo, "a priori" la publicidad tampoco tendría cabida como financiadora de medios de comunicación; ya que se apuesta por los llamados medios comunitarios y se propone que debe haber una apropiación de los medios y de sus contenidos por parte de la comunidad y no una mercantilización de los mismos, lo que en principio parece contradictorio con su explotación publicitaria.

Sin embargo, sí hay algunos ejemplos de experiencias de comunicación participativa en los que la publicidad está presente, ya sea como fuente financiadora o en forma de mensajes de interés público y social que se divulgan en medios comunitarios de forma gratuita. En especial en la radio, que ha demostrado ser el medio de mayor tradición y potencial para generar cambios sociales desde la comunicación participativa.

Es así, en los casos de: Radio Quillabamba en Perú (donde la publicidad comercial se utiliza para los gastos de infraestructura como salarios, oficinas, teléfono y electricidad; pero está regulada y limitada a ciertas horas y días de la semana, mientras que la publicidad social se transmite sin coste); Radio Izcanal en El Salvador, (que difunde avisos publicitarios de pequeñas empresas comerciales, dirigidos a la población local y donde los mensajes de contenido educativo son también numerosos e importantes); Radio Zibonlele en Sudáfrica (que desde 1996

4 Gumucio, A. (2001), Haciendo Olas. Historias de comunicación participativa para el cambio social. Fundación Rockefeller, p.6. Disponible en

http://www.communicationforsocialchange.org/pdf/haciendo_olas_[f]_7_23.pdf [consultada el 1 de abril de 2012] 
es auto sostenible financieramente en parte a través de los ingresos percibidos por la publicidad, y difunde mensajes sobre atención primaria de la salud que contribuyen a mantener a la comunidad informada y sana); Radio Chaguarurco en Ecuador (entre cuyas fuentes de ingreso se incluyen la publicidad en un $20 \%$ del ingreso y los avisos comunitarios en un 40\%: Radio Mampita y Radio Magneva en Madagascar (que se autofinancian parcialmente con publicidad y con la contratación de espacios de programación por parte de las agencias de cooperación internacional sobre temas de medio ambiente o de salud); o Bush Radio en Ciudad del Cabo (financiada en parte por publicidad, pero que rechaza los anuncios de bebidas alcohólicas, debido a que el alcoholismo es uno los problemas que enfrenta la comunidad $)^{5}$.

También hay casos que pueden considerarse buenos ejemplos de comunicación participativa en los que la publicidad está presente, además, como herramienta de difusión de mensajes sociales en manos del agente que encabeza el proyecto o como promotora de la participación comunitaria en él. Así, en el proyecto de promoción de la salud Radio Soul City en Sudáfrica, se aprovecha el potencial de los medios masivos para inducir cambios sociales y se incluye una estrategia de relaciones públicas y publicidad con una función doble: divulgar las series de TV y radio, que ellos mismos producen, y llamar la atención sobre ciertos tópicos de salud. O en la Carpa Lila, en Bolivia, cuarta fase de un ambicioso programa de mejora de la salud reproductiva en el largo plazo, en el que la sistematicidad en la realización de campañas a través de los medios masivos (y de la publicidad) ha conseguido buenos resultados; y se ha sabido evolucionar hacia un enfoque más participativo para poner en marcha una estrategia colectiva capaz de promover el análisis y el debate sobre el tema en relación con los intereses de la comunidad. Además de la pieza central (una carpa en la que se desarrollan todo tipo de actividades y que se desplaza por el país), se han utilizado otros medios de difusión como interesantes folletos explicativos especialmente dirigidos a los jóvenes.

A juzgar por los ejemplos aquí mencionados, la publicidad no está, como decíamos al principio, totalmente ausente en proyectos de desarrollo y cambio social centralizados en estrategias de comunicación participativa.

Aunque sí parecen existir frenos para su utilización en este tipo de proyectos, debido sobre todo a su estrecha vinculación con los modelos de corte difusionista y a que es entendida desde un esquema tradicional como una forma de comunicación: vertical (en la que el beneficiario es mero receptor de mensajes y los decisores de la estrategia están muy lejos); masiva (vinculada con los grandes medios masivos más que con los medios comunitarios); impositiva y manipuladora (se trata de imponer un sentido a través de un mensaje único que no admite réplica); objetivista (busca resultados en función de unos objetivos previamente marcados para la causa y para la entidad); exógena (desde parámetros alejados al contexto y a la cultura de los beneficiarios); costosa (se asume que es necesaria una inversión elevada para hacer una gran campaña de publicidad); etc.

5 Ver todas estas experiencias en Gumucio, A. (2001), op.cit. 
De este modo, la influencia que todavía tienen las teorías modernizadoras, instaladas en la mentalidad de numerosos organismos responsables de programas de cambio social, y la extensión y consolidación del marketing social como técnica al servicio de los más diversos emisores con fines sociales (tanto en programas de desarrollo como en los decadentes estados del bienestar), hace que dentro del marco actual de la comunicación para el cambio social, la publicidad se siga considerando imprescindible para:

- La difusión masiva de determinadas actitudes y conductas que permitan generar en una comunidad los cambios que se consideran necesarios para lograr una mejora, especialmente en el ámbito de la salud. Partiendo siempre de la idea de que la solución a un determinado problema está vinculada con un cambio comportamental.

- La recaudación de fondos, ya sea que estén destinados a financiar la labor cotidiana de determinadas instituciones, como a solventar necesidades puntuales surgidas de catástrofes.

- La financiación de los medios de comunicación, tanto de los ya existentes en un contexto como de los que puedan crearse.

- Dar visibilidad a determinados problemas y a sus soluciones, o mejor, a las soluciones que en cada caso, en cada contexto, traten de imponerse como las adecuadas para paliarlo.

- Promover la participación de las personas de una comunidad en un determinado programa.

- Dar notoriedad y construir la imagen de los emisores de todo tipo (estados, fundaciones, organizaciones no gubernamentales, organismos internacionales, etc., que ejercen como anunciantes), en un contexto cada vez más competitivo y saturado de entidades con fines sociales y que, con independencia de los problemas y las causas que atienden, necesitan notoriedad y credibilidad para subsistir.

Y para ello sigue estando hoy demandada de forma mayoritaria desde los agentes responsables del desarrollo y la acción social en el mundo; dando incluso a veces la sensación de que se acude a ella como por inercia, como un recurso imprescindible y socorrido; como si por el hecho de hacerla se solucionara de algún modo el problema.

Así es vista desde el marco del desarrollo y el cambio social, como un instrumento más al servicio de otros fines. Pero ¿Cómo ha percibido la publicidad, entendida como sistema, como profesión, como industria o como institución la necesidad de cambio o desarrollo social? ¿Cuáles han sido sus aportaciones a este territorio?

\section{EL DESARROLLO Y EL CAMBIO SOCIAL DESDE LA PERSPECTIVA DE LA PUBLICIDAD}

No hay duda de que, desde el origen mismo de la disciplina publicitaria ha existido en sus profesionales más prestigiosos una fuerte inquietud, por evidenciar y 
aprovechar la el poder de la publicidad para el cambio y la mejora de las sociedades. Así lo manifestaron en sucesivas épocas grandes hombres del pensamiento y la práctica publicitaria como fueron Prat Gaballí en España, David Ogilvy en Gran Bretaña o Howard Gossage en Estados Unidos; para los que su mayor satisfacción profesional fue contribuir a través de la publicidad a grandes causas como la higiene, la lucha contra el cáncer o la protección del medioambiente.

Y más recientemente otros muchos, a través de iniciativas tan distintas como la polémica exhibición de temas sociales realizada a través de la publicidad gráfica encabezada por Oliviero Toscani en la década de 1990 en Italia, o la fundación de la red online Publicitarios sin Fronteras por iniciativa del argentino Guillermo Caro.

Quizás sea por la incómoda conciencia latente en el corazón de todos aquellos que se han dedicado profesionalmente a la publicidad, y/o por la firme creencia en que su poder no podía desaprovecharse sólo con causas comerciales. Pero lo cierto es que en sus últimos cien años de existencia la industria publicitaria ha generado, no sólo incontables mensajes destinados a todo tipo de causas sociales, sino también iniciativas más estables que han tratado de sistematizar este tipo de publicidad.

Podemos decir que existe una historia doble de esta modalidad publicitaria. Por un lado estaría la historia de los anuncios o avisos con fines sociales, que en sus antecedentes podría remontarse incluso al siglo XVI, y que continúa en nuestros días. Esta historia, que comienza cuando el término publicidad todavía no tiene las connotaciones comerciales actuales, incluiría anuncios de interés público o social realizados esporádica o puntualmente para satisfacer una demanda social concreta. Como por ejemplo los que se hacen todavía hoy para recaudar fondos ante catástrofes naturales o los que demandan a la industria de la publicidad entidades minoritarias con poco o ningún presupuesto que necesitan dar un impulso a su causa y a su labor. En este caso, existe la costumbre por parte de las agencias de publicidad de atender de forma benéfica este tipo de tareas, es decir, de asumir los costes derivados del trabajo creativo y de producción.

Pero hay una historia propia de la publicidad social, entendida de forma sistemática. Esta se inicia después de la Segunda Guerra Mundial, tras la prueba de fuego que supone para la publicidad ponerse por segunda vez al servicio de las diversas demandas de una causa tan dramática como: el reclutamiento de soldados, el apoyo moral, el ahorro de bienes escasos o la recaudación de fondos ${ }^{6}$. Y esta vez será definitiva, ya que dará paso a la sistematización de este tipo de publicidad al servicio de causas sociales por parte de distintos emisores, tal como la conocemos hoy y como la hemos descrito antes al hablar de su vinculación con el marketing social.

Surgen los que serán grandes emisores de este tipo de publicidad, organismos internacionales, organizaciones no gubernamentales, fundaciones medioambientales o gobiernos democráticos; pero también instituciones propias del sistema publicitario especializadas en coordinar y difundir este tipo de publicidad como el Ad

6 Para un estudio más completo de la perspectiva histórica y estructural de la publicidad social ver Alvarado López, M.C. (2010): La publicidad social, una modalidad emergente de comunicación. Universidad Complutense de Madrid, Tesis doctoral dirigida por Raúl Eguizábal Maza y leída en diciembre de 2003. Edición digital disponible en http://eprints.ucm.es/11522/ 
Council en los Estados Unidos (secuela del War Advertising Council que funcionó ya en 1942), el Consejo Publicitario Argentino (1960) o la Fundación Pubblicitá e Progresso en Italia (1975).

Por destacar un caso concreto, podemos citar aquí la contribución social realizada históricamente por el que se puede considerar medio publicitario por excelencia, el cartel. Son innumerables las ocasiones en que se ha utilizado (y se sigue haciendo) el cartel al servicio de causas sociales (contra el racismo, por el sufragio femenino, recaudatorio, cartel pacifista, en pro de los derechos humanos, etc.), y también políticas. Tanto es así que recientemente, John Downing ha mencionado los carteles anarquistas, socialistas y marxistas realizados en España antes de 1939 como ejemplo de lo que llama nano-medios de comunicación ${ }^{7}$.

Si bien, como ya se ha mencionado, una parte de este esfuerzo publicitario se ha realizado y se sigue realizando gratuitamente, es decir, de forma benéfica (sobre todo en los casos de colaboraciones puntuales con entidades de escaso presupuesto); la tendencia es a una profesionalización cada vez mayor de este sector. De hecho existen agencias de publicidad especializadas en marketing y publicidad social y festivales internacionales específicos en los que concursa; pero también bibliografía e investigaciones que abordan el tema cada vez en mayor profundidad.

Es sin duda un sector en alza que incluso está demandando profesionales de la comunicación, tanto para las entidades sociales, como para las empresas intermediarias.

Por tanto, no se puede negar que el sistema publicitario ha sido siempre consciente de su poder de cambio social y ha ofrecido su maquinaria: su técnica, su lenguaje y su saber, al servicio de numerosas causas: catástrofes inesperadas, ambiciosos proyectos de desarrollo, programas de prevención sanitaria de calado mundial, etc. Y ello a pesar de sufrir sucesivas crisis que no parecen terminar nunca y de estar en permanente reconstrucción y expiación.

Sin embargo, no podemos dejar de reseñar aquí algunas cuestiones que merecen una reflexión crítica sobre el papel ejercido por la industria publicitaria en relación con su repercusión en la mejora de la sociedad:

Se ha generado una nueva modalidad publicitaria (publicidad social, con fines sociales, solidaria, de interés público, de bien común etc.), que se ha utilizado tanto en programas de desarrollo llevados a cabo en países y comunidades con graves necesidades de mejora, como en las sociedades desarrolladas bajo los parámetros del estado del bienestar. Pero que se ha entendido e implementado desde los esquemas de mercado y beneficio que impone la publicidad comercial.

El grueso de la industria ha seguido trabajando mayoritariamente para causas comerciales, siendo la publicidad social escasa y relegada a una menor difusión en los grandes medios masivos por la escasez de presupuestos.

7 Downing, J. (2010), Nanomedios de comunicación: ¿Medios de comunicación comunitarios ?¿O de red? ¿O de movimientos sociales? ¿Qué importancia tienen? ¿Y su denominación?; texto para la conferencia "Medios comunitarios, movimientos sociales y redes", organizado por la Cátedra Unesco de Comunicación InCom-UAB en colaboración con la Fundación CIDOB, Barcelona, 15/03/2010. Disponible en http://www.portalcomunicacion.com/catunesco/download/2010_DOWNING_NANOMEDIOS\%20DE\%20 COMUNICACI\%D3N.pdf [consultado el 10 de diciembre de 2012] 
- Este tipo de publicidad ha servido como escaparate para los creativos publicitarios, que han ganado muchas veces premios en prestigiosos festivales con este tipo de anuncios; y también como una forma de expiación de toda la industria frente a las críticas que la hacen responsable de las lacras del capitalismo.

- También ha servido como distractor de los problemas reales, haciéndonos creer que, por hacer publicidad, el problema ya quedaba en parte resuelto; o que, con una mera acción puntual (a veces mediada por el consumo) éramos más solidarios y humanos.

- La dominancia en ocasiones de un enfoque caritativo o benéfico, no ha favorecido la estabilidad de las campañas y de las temáticas sociales, ni el largo plazo que muchas veces estas temáticas precisan.

- En pro de la notoriedad, se han primado los enfoques negativos y emocionales de determinados temas sociales (hambre, salud, accidentes de tráfico, violencia de género, etc.); y se han potenciado algunos estereotipos negativos (como el de África a través de la imagen del niño famélico y moribundo); lo que en ocasiones ha generado rechazo, no ha favorecido cambios estables de actitud y ha alejado a los receptores de la realidad.

- Además, la publicidad social ha contribuido a construir en los receptores un imaginario compartido sobre las lacras de nuestra sociedad, influyendo en nuestro modo simplificado y distante de entender los problemas y sus soluciones.

- Se ha dado por hecho que por ser para causas sociales este tipo de publicidad era beneficiosa e incluso éticamente "buena", sin llevarse a cabo investigaciones rigurosas sobre sus consecuencias más allá de sus resultados inmediatos.

- La existencia de esta modalidad publicitaria ha evitado quizá un cambio de toda la industria hacia la asunción de una responsabilidad social que, por encima de las causas a las que sirve, tiene como institución social. Se ha olvidado que el cambio tiene que ser de toda ella.

Resumiendo este apartado, podemos afirmar que la aportación fundamental de la industria y del sistema publicitario al desarrollo es la de haber dado a luz una publicidad específicamente social que, desde los parámetros del mercado, se ha utilizado como instrumento a demanda de muy diversos emisores y cuyo impacto global, con independencia de la eficacia obtenida en cada caso, resulta hoy muy difícil de evaluar.

Pero ¿es eso todo lo que puede hacer por la mejora de la sociedad una forma de comunicación a la que, paradójicamente, consideramos cómplice de la devastadora crisis económica que padecemos?

Quizá, bajo el paradigma de la nueva comunicación para el cambio social, la publicidad pueda hacer algo más, aunque para ello sea la primera que deba cambiar. Veamos cómo.

\section{LA NUEVA PUBLICIDAD EN LA COMUNICACIÓN PARA EL CAMBIO SOCIAL}

Como hemos visto antes, la publicidad, entendida sólo y exclusivamente como sistema mercantil, como comunicación vertical, persuasiva, masiva, pagada, intere- 
sada, etc., no parece encajar con los modelos de cambio derivados de las teorías de la dependencia, desde los que se precisa una comunicación alternativa y un nuevo perfil de comunicador.

Pero tampoco parece hacerlo en el contexto de la sociedad actual, cada vez más crítica y convulsionada gracias a los cambios radicales que en todos los ámbitos (económico, político, tecnológico, mediático, etc.), se suceden a gran velocidad. En especial con la revolución que parece prefigurar el acceso a los nuevos medios digitales y a las redes sociales que ha propiciado la rápida introducción en nuestras vidas de internet.

Según los expertos en comunicación para el cambio social, Alfonso Gumucio y Thomas Tufte, esta podría definirse como

un proceso de diálogo y debate basado en la participación y acción colectiva, a través del cual la propia gente determina lo que necesita para mejorar sus vidas. En el corazón del concepto está la convicción de que las comunidades afectadas entienden mejor su realidad que los "expertos" ajenos a ella ${ }^{8}$.

¿Puede la publicidad formar parte de este proceso?

Para ello tendría que prestarse al diálogo con aquellos a los que va a dirigirse, ponerse por entero en sus manos, no imponerse desde fuera sino decidirse, pensarse y hacerse desde y con la participación de la comunidad afectada, en el caso de que ésta lo considere necesario para su mejora.

Esto supone ir mucho más allá de donde se ha ido hasta ahora a través de una publicidad social algo que, si tenemos en cuenta los cambios que la publicidad está experimentando actualmente no parece imposible.

Si como afirman también Gumucio y Tufte, la expresión "mercadeo social" encierra un oxímoron ${ }^{9}$, para el profesor Antonio Caro hablar de publicidad solidaria o social también, y hay para ello numerosas razones.

Según Caro: "El modelo dominante de publicidad no es solidario o si lo es, lo es de forma disfrazada..." "...La publicidad no es un instrumento neutro y constituye el instrumento por antonomasia del capitalismo desde el que organizar la cohesión social...", y “...en manos de de las entidades sin ánimo de lucro tiende a mercantilizar éstas" 10 .

Para Caro la única salida sería una vuelta atrás, es decir, despojar a la publicidad de toda connotación comercial y devolverle su acepción comunicacional más pura la que hace referencia a publicitar en el sentido de "hacer algo público".

Y esta propuesta de que la publicidad sea más comunicación y menos publicidad no parece descabellada y menos en el territorio de lo social.

8 Gumucio, A. y Tufte, T.,(comps.), (2008) Antología de comunicación para el cambio social, lecturas históricas y contemporáneas, Consorcio de Comunicación para el Cambio Social, Plural Editores, La Paz, Bolivia, p.23.

9 Gumucio, A. y Tufte, T.,(comps.), (2008), op.cit. p.20.

10 Afirmación realizada por el profesor Antonio Caro Almela en una de las sesiones del Máster en Comunicación con Fines Sociales que se imparte en el Campus de Segovia de la Universidad de Valladolid, el día 27 de enero de 2012. 
En primer lugar hay que decir que la publicidad está sufriendo en estos momentos una revolución radical que la está haciendo virar hacia el consumidor, hacia la sociedad y hacia sí misma.

La crisis mundial, los avances tecnológicos y especialmente los acaecidos en el panorama de los medios, la explosión de las redes sociales, los cambios en los consumidores y en la sociedad, etc., están haciendo que, por su propia supervivencia, los teóricos y los profesionales de la publicidad estén reflexionando más que nunca sobre estas posibilidades de cambio.

Se habla ya de una postpublicidad ${ }^{11}$, pero también de publicidad una conversada (o conversacional); lo que de alguna manera podría entenderse como una paradoja aún mayor que las anteriormente citadas.

En un contexto donde los consumidores y los ciudadanos pueden, a través de la comunicación digital, manifestar sus opiniones de campañas, productos, empresas y marcas de un modo constante, directo y crítico; es decir, interactuar con los emisores, no hay otra salida para la actividad publicitaria que evolucionar.

Parafraseando de nuevo a Caro

Conforme la publicidad —o la comunicación — interactiva vaya desplazando a la publicidad unidireccional característica de los medios masivos, más la publicidad conversacional tenderá a desplazar a aquella publicidad basada en el monopolio de la palabra de que han gozado, desde los inicios mismos de la actividad publicitaria, anunciantes y publicitarios. Una publicidad ya no dirigida en lo básico a imponer por parte del anunciante el comportamiento que debe seguir el consumidor en función de los fines que aquél persigue, sino que habrá de dialogar con este... ${ }^{12}$.

Una de las consecuencias de este cambio es, como vemos, la puesta en cuestión del concepto de publicidad dominante hasta ahora, algo que en esta disciplina es una constante.

Pero más allá de la cuestión conceptual, nos interesa aquí centrarnos en esa posibilidad de diálogo que apuntan tanto profesionales como teóricos de la publicidad.

Las perspectivas de que la publicidad interactiva vaya cada vez más sustituyendo a la publicidad unidireccional, es decir, a la publicidad tradicional (tal como la hemos entendido hasta ahora) concluirían, por tanto con una nueva publicidad a la que podemos llamar conversacional o también dialógica.

Si el requisito para que exista este tipo de publicidad pasa por propiciar la interacción con aquellos a los que va a dirigirse, y a pesar de que este cambio radical parezca venir propiciado por las nuevas tecnologías, no tiene por qué ser así. Y menos aún en el caso de una publicidad con fines sociales en el marco de la comunicación para el cambio social, donde el diálogo directo entre las personas de una

\footnotetext{
11 Ver la reflexión que ofrece al respecto el planteamiento que hace el publicitario español Daniel Solana en su libro Postpublicidad disponible en versión líquida en http://www.postpublicidad.com/

12 Caro, A. (2012): "El futuro de la publicidad y la (necesaria) vuelta a lo básico", declaraciones para la web www.marketingdirecto.com con motivo de su participación en el congreso The future of advertising, organizado por la mencionada web en Madrid, en marzo de 2012. Ver en http://www.marketingdirecto.com/especiales/the-future-of-advertising-2012/antonio-caro-el-futuro-de-lapublicidad-y-la-necesaria-vuelta-a-lo-basico/ [consultado el 10 de abril de 2012]. Ver también otras ideas de este autor en la obra Comprender la Publicidad.
} 
determinada comunidad y los planificadores de la acción o el proyecto social es, como hemos visto, la base.

Creemos, por tanto, que puede existir una publicidad dialógica en aquellos proyectos de cambio social en los que la comunidad determine que la publicidad puede contribuir a la mejora del problema en cuestión. Una decisión que, evidentemente puede ser también debatida con los planificadores, pero que debe surgir de la comunidad y nunca estar preestablecida de antemano por éstos.

En caso de que se decida que la publicidad sea una de las herramientas a emplear, el proceso de decisión y elaboración de la acción concreta a llevar a cabo deberá también ser participativo. En él deben formar parte personas de la comunidad beneficiarias del proyecto, expertos en comunicación de agencias o empresas locales y también de la entidad promotora del mismo.

Este diálogo debe servir para determinar el "qué" y el "cómo", es decir, lo que hay que comunicar en los mensajes y a través de que lenguaje y de qué medios hacerlo; teniendo en cuenta la cultura y el contexto en el que van a funcionar, de lo que es precisamente una garantía el diálogo.

Para ello será necesario que los expertos y profesionales de la comunicación se olviden de la verticalidad y de la jerarquía que es habitual en las relaciones internas entre anunciante-agencia de publicidad, y entiendan que lo importante en estos casos no es ganar premios en prestigiosos festivales, sino participar y facilitar un proceso de cambio desde la horizontalidad y desde el diálogo.

También es factible la participación de la comunidad en los procesos de materialización de las acciones y piezas comunicativas, que podrá ser más o menos artesanal o profesional, en función de las prioridades y de los recursos asignados. Y en su difusión, para la que, por ejemplo, los medios comunitarios o las expresiones culturales propias podrían ser importantes.

De este modo, no es la publicidad un instrumento que sirve a un determinado programa cambio, sino un proceso potencialmente generador de ese cambio.

Queda claro en esta propuesta que entendemos que es posible la integración de una publicidad dialógica en la comunicación para el cambio social en cualquier tipo de contexto, incluso en el de las sociedades desarrolladas ${ }^{13}$.

Pero, además, y dado que el paso a una publicidad dialógica parece un proceso largo, si no una utopía, trataremos de ofrecer algunas claves más que pensamos pueden favorecer y mejorar la participación de la publicidad en la comunicación para el cambio social.

\footnotetext{
13 Así ha sido en la experiencia de formación-investigación-acción que se está llevando a cabo desde hace siete años en la ciudad de Segovia, de la mano de los profesores Rodrigo González y Susana de Andrés, con el objetivo de favorecer la inclusión de las personas con discapacidad mental, en la que estas personas, con la mediación de la entidad FEAPS Castilla y León, trabajan conjuntamente con alumnos de la licenciatura de Publicidad y RR.PP., (de la Facultad de CC. Sociales, Jurídicas y de la Comunicación de la Universidad de Valladolid en el campus de Segovia) para la elaboración de carteles con la finalidad de sensibilizar a la población; pero generando un proceso que sensibiliza también a los futuros profesionales. Ver en Andrés del Campo, S. (de) y González Martín, R. (2011): “Comunicación inclusiva. Una experiencia en creación de campañas sobre discapacidad intelectual". Área Abierta. N. ${ }^{\circ} 31$, Diciembre 2011.
} 
En primer lugar, creemos que es necesaria una toma de conciencia de la responsabilidad que tiene la actividad publicitaria en su conjunto sobre la sociedad, y de que puede y debe formar parte del cambio que se está demandando globalmente. Y no sólo desde ese nuevo modelo de publicidad conversada o dialogada, o desde una publicidad social, sino desde cualquiera de sus manifestaciones más o menos tradicionales, en cualquier medio o soporte y al servicio de cualquier tipo de anunciante.

La publicidad debe ofrecerse para propiciar el cambio a través de su dimensión estratégica, su potencial lúdico, de una creatividad responsable y de una apuesta definitiva por la responsabilidad y la ética; ser capaz de generar discursos sobre los grandes temas del desarrollo y del cambio social.

Se necesita para ello un mayor cuestionamiento teórico de la comunicación publicitaria por parte de todos los actores implicados en ella, desde el que la consideración de sus efectos culturales y sociales y de su función ideológica e institucional sea el eje.

La publicidad, asumida como lenguaje social dominante en nuestro mundo globalizado es continuamente parodiada por receptores anónimos, y criticada, en especial por los grupos anti-sistema, a través de la llamada contra-publicidad. Es por ello que se necesita un mayor nivel de reflexión y autocrítica desde dentro del sistema mismo que propicie un cambio real y no un simple lavado de imagen.

Para ello sería necesario establecer como una prioridad la mejora de la formación de los futuros profesionales de los grados en comunicación, algo que en el contexto español se ha intentado con desigual resultado en el diseño de los nuevos grados para su adaptación al Espacio Europeo de Educación Superior.

Además, es necesaria una formación específica que satisfaga la demanda de profesionales de la comunicación para el cambio social. Un perfil complejo, que suele cubrirse con profesionales procedentes del periodismo, pero que también podrían proceder de una formación en publicidad, dado que cada vez más, se necesita una doble visión estratégica y creativa que los grados en Publicidad y RR.PP., suelen aportar $^{14}$. Entre los rasgos esenciales que definen este perfil y como muy bien señala Gumucio $^{15}$, no pueden faltar los relacionados con esa dimensión participativa y dialógica, como el asumir un rol de servicio, ser facilitador de procesos y promover el diálogo y el debate. La predisposición a conocer la cultura y las características de la comunidad o colectivo en el que se va a trabajar, saber escuchar lo que las personas tienen que decir y demostrar respeto por sus ideas y actitudes; son cualidades imprescindibles en este nuevo modelo con independencia del terreno en el que se vaya a trabajar, ya sea que se pretenda mejorar la salud en una aldea boliviana o favorecer la inclusión de las personas con discapacidad en la sociedad española.

14 Existen ya en España algunas respuestas formativas en las que este enfoque tiene peso, entre ellos cabe citar aquí: el Máster Universitario Internacional en Estudios de Paz, Conflictos y Desarrollo de la Universitat Jaume I (UJI) de Castellón en el marco de la Cátedra UNESCO de Filosofía para la Paz, el Máster en Comunicación y problemas Socioculturales de la Universidad Rey Juan Carlos; o el Máster en Comunicación con Fines Sociales: Estrategias y Campañas, que se imparte en el Campus de Segovia de la Universidad de Valladolid.

15 Gumucio Dragón, A. (2004), op.cit., pp. 19-21. 
Sería necesario también documentar y divulgar las experiencias de comunicación para el cambio social en las que, más allá de su función como fuente de ingresos de determinados medios comunitarios, como promotora de la participación o difusora de un determinado mensaje a la población, la nueva comunicación publicitaria, tal como aquí se ha tratado de esbozar, sea considerada de un modo dialógico como parte de una estrategia comunicacional de cambio.

No parece, pues, que haya razones de peso para excluir a la publicidad de los programas de comunicación para el cambio social. Las inercias generadas por el difusionismo, los prejuicios negativos o el desconocimiento parcial por parte de los planificadores sociales, no debería mermar las posibilidades que, más allá de su uso tradicional, y en este nuevo giro hacia el diálogo, podría tener como valiosa aliada.

Y los planificadores del cambio social deben verlo.

\section{REFERENCIAS BIBLIOGRÁFICAS}

Aguirre Alvis, Jose Luis y Condori, M.b (2000): Las radios comunitarias en Bolivia: despojados de todo, incluso de la palabra, Presencia Juvenil, la Paz, Bolivia.

Alvarado López, María Cruz (2010): La publicidad social, una modalidad emergente de comunicación. Universidad Complutense de Madrid, Tesis doctoral dirigida por Raúl Eguizábal Maza y leída en diciembre de 2003. Edición digital disponible en http://eprints.ucm.es/11522/

Andrés Del Campo, Susana y González Martín, Rodrigo (2011): "Comunicación inclusiva. Una experiencia en creación de campañas sobre discapacidad intelectual”. Área Abierta. n. ${ }^{\circ}$ 31, Diciembre 2011.

CARO, Antonio (2012): "El futuro de la publicidad y la (necesaria) vuelta a lo básico", declaraciones para la web www.marketingdirecto.com con motivo de su participación en el congreso The future of advertising, organizado por la mencionada web en Madrid, en marzo de 2012

http://www.marketingdirecto.com/especiales/the-future-of-advertising-2012/antoniocaro-el-futuro-de-la-publicidad-y-la-necesaria-vuelta-a-lo-basico/

- (2010): Comprender la publicidad, Ed. Trípodos, Colección Extensiones, Facultad de Comunciación Blanquerna, Universitat Ramón Llull, Barcelona redalyc.uaemex.mx/src/inicio/ForazarDescargaArchivo.jsp?...

[consultado el 10 de abril de 2012]

DownING, J. (2010): Nanomedios de comunicación: ¿Medios de comunicación comunitarios? ¿O de red? ¿O de movimientos sociales? ¿Qué importancia tienen? ¿Y su denominación?; texto para la conferencia "Medios comunitarios, movimientos sociales y redes", organizado por la Cátedra Unesco de Comunicación InCom-UAB en colaboración con la Fundación CIDOB, Barcelona, 15/03/2010. Disponible en http://www.portalcomunicacion.com/catunesco/download/2010_DOWNING_NANOMEDIOS\%20DE \%20COMUNICACI\%D3N.pdf [consultado el $1 \overline{0}$ de diciembre de 2012]

Gumucio Dragón, A. (2004): "El cuarto mosquetero: la comunicación para el cambio social", en Investigación y Desarrollo, col. 12, n. ${ }^{\circ} 1$ (2004) pp. 2-23. (Documento presentado al VI congreso de la Asociación latinoamericana de Investigadores de la Comunicación- ALAIC, realizado en Santa Cruz de la Sierra, Bolivia, del 5 al 8 de junio de 2002). Disponible en http://ciruelo.uninorte.edu.co/pdf/invest_desarrollo/121/el_cuarto_mosquetero.pdf [consultada el 10 de octubre de 2011] 
Gumucio, A. (2001): Haciendo Olas. Historias de comunicación participativa para el cambio social. Fundación Rockefeller, p.6. Disponible en http://www.communicationforsocialchange.org/pdf/haciendo_olas_[f] 7 23.pdf [consultada el 1 de abril de 2012]

(1999): Comunicación, desarrollo y participación, presentación disponible on line en http://www.slideshare.net/Gumucio/comunicacin-desarrollo-y-participacin, [consultada el 15 de febrero de 2012]

Gumucio, A. y TufTe, T.,(comps.), (2008): Antología de comunicación para el cambio social, lecturas históricas y contemporáneas, Consorcio de Comunicación para el Cambio Social, Plural Editores, La Paz, Bolivia, p.23.

http://recursostic.javeriana.edu.co/cyl/syp/components/com_booklibrary/ebooks/5818V aca.pdf [consultado el 10 de febrero de 2011]

Solana, D. (2010): Postpublicidad, Ed. Índice Arts Grafiques, Barcelona. Disponible en versión líquida en www.postpublicidad.com

VAca Gutiérrez, H. (2011): "Procesos interactivos mediáticos de Radio Sutatenza con los campesinos de Colombia (1947-1989)", en Signo y Pensamiento, n. ${ }^{\circ}$ 58. Documentos de Investigación, pp.254-269, volumen 30, enero-junio 2011, disponible en

\title{
RESUMEN
}

La reflexión sobre la publicidad en el marco de la investigación-acción de la comunicación para el desarrollo, ha sido con frecuencia olvidada o contemplada de un modo menor; quizás por ser entendida sólo como una herramienta necesaria del marketing social, supeditada a los programas de desarrollo de corte difusionista que, a pesar de la crítica y de los nuevos modelos hoy vigentes, todavía subsisten.

En el contexto reciente y de futuro, en el que, guste o no, gana fuerza la etiqueta "comunicación para el cambio social", la publicidad, entendida como una institución social y globalmente legitimada tiene reservado un papel trascendental que, dados los retos de mejora y las necesidades urgentes de la nueva sociedad mundial, los profesionales e investigadores debemos saber ver y aprovechar.

Palabras clave: publicidad, desarrollo, comunicación, cambio social.

\begin{abstract}
We present in this paper a reflection on advertising within the framework of the action- research of communication for development, a subject that has often been forgotten or covered in a minor way; perhaps being understood only as a necessary tool of social marketing, subject to a "diffusionist" point of view of development programmes in spite of the criticism and the incoming new models. In the context of recent times and future, that inevitably give strength to the label "communication for social change", the advertising area, understood as a social institution and globally legitimized, has reserved a fundamental role which, given the challenges of improving society and the urgent needs of the new global society, practitioners and researchers need to know and exploit.
\end{abstract}

Key words: advertising, development, communication, social change. 


\section{RÉSUMÉ}

Réflexion sur la publicité dans le contexte de la recherche-action de la communication pour le développement, laquelle a été souvent oubliée ou visée dans un mode mineur ; peut-être comprise comme un outil nécessaire du marketing social, soumis au point de vue diffusioniste que les programmes de développement ont pris en dépit de la critique, ce que nous forcé aujourd'hui au développement de nouveaux modèles. Dans le contexte de ces dernières années et de l'avenir, dans lesquels prit de plus en plus forcé l'etiquette « communication pour le changement social», la publicité, comprise comme une institution sociale et légitimé dans le monde entier aura un rôle mémorable que, devant les défis de l'amélioration de la vie sociale et les besoins urgents de la société mondiale, doivent les praticiens et les chercheurs savoir voir et exploiter.

Mots clé: publicité, développement, communication, changement social. 